\title{
IMAGE COMPRESSION USING LOCAL MAP VIEW TECHNIQUE
}

\author{
C Rajeswari \\ Research Scholar, Department of Computer Science \& Applications, \\ SCSVMV University, Kanchipuram, TN, India \\ rajic13@gmail.com \\ Dr.S.Prakasam \\ Associate Professor, Department of Computer Science \& Applications, \\ SCSVMV University, Kanchipuram, TN, India \\ sp@kanchiuniv.ac.in
}

\begin{abstract}
:
Image compression has gained importance in many medical treatments and diagnostic processes. Image compression aims at correctly separating different tissues, organs, or pathologies in volumetric image data. Most of the existing algorithms for Image compression have a "scattered" cluster problem (disconnected clusters) happened in many clustering techniques (agglomerative, k-means, Dbscan) above algorithms not taken into account of both quality value and connectivity of points and region varying shapes. This proposed technique LMV (Local Map View) concentrates on determination of local quality for each point in all instances of the region in the comparative similarity view by applying the initial cluster technique. This view allows the user to choose instances for detailed analysis and filter the outlier instances from the input, next specific feature selection process identifies regions with systematic characteristics across the images. Then the process continues showing threshold marked of the regions and displays the Image compression quality across selected instances for standard image values as resultant dataset. This proposed technique shown the better results in case Image compression constraints (multi-level threshold, MSE, FRR) by the existing techniques.
\end{abstract}

\section{KEYWORDS: Images, LMV, MSE, FRR, Image compression.}

\section{Introduction}

Image division for the most part includes characterizing mammograms into a few areas, including the bosom fringe [4], [9], the areola [8] and the pectoral muscle. The important part on an image is the breast outskirt, also called the skin-air interface, or bosom limit. The breast form can be acquired by compression in the [15] imageinto breast and non-breast areas. The extracted breast contour should adequately model the soft tissue/air interface and preserve the nipple in profile.

\section{A. Edge Detection Methods:}

Edge identification is a basic field in the zone of picture taking care of. Since there is a sharp change in power in as far as possible, as far as possible and edges are immovably related. The base of another division technique is edge area framework. Michael A. Wirth [16], [14] depicts the use of dynamic shapes. The dynamic shapes can be utilized to extricate the bosom district in mammograms. Breast shape is one of the biggest single highlights of mammogram. [15] It is also called skin air interface. Bosom frame lies amidst the sensitive tissue and the non-chest locale. Extraction of the chest shape licenses finding the inconsistencies in the region of the bosom.

\section{B. Image Enhancement Analysis:}

Organic examinations affirm that bosom malignant growth is the after effect of an aggregation of an extensive number of individual hereditary transformations that on the whole adjust components of the complex inside flagging arrangement of a cell. Ceaseless replication of a ruined cell goal as territory of strange cells that [1] may collect other abnormal changes to in the end start malignant growth. What causes these transformations has been the subject of discussion over various years, but since such a significant number of hereditary adjustments are included, it is presently yielded that one single factor could not in any way, shape or form start every one of the progressions. 


\section{Fusion Methods:}

Fusion methods can be broadly classified into three categories: (i) voting-based methods [5],[19],[25],[23] (ii) distance-based methods [23], [13], [18], and (iii) statistically driven methods [26],[3],[6],[2],[23] Voting-based methods assign a weight to the decisions made by each template regarding the probable output label at each voxel in the target image, and finally select a label that satisfies certain optimal criteria. Distance-based methods [7],[11],[10],[23] compute the signed Euclidean distances to the contours of the structures, weigh those distances based on the similarity information, and finally assign a label that results in the least cumulative weighted Euclidean distance.

\section{Stable Technic:}

Simultaneous Truth and Performance Level Estimation (STAPLE) is a widely used algorithm [5] that belongs to the third category of statistical fusion methods. The STAPLE algorithm not only generates the output Image compressions (or reference standard), but also simultaneously rates the performances of the input Image compressions. In practice, there are especially two specific scenarios [23] where the STAPLE algorithm is widely used. First, it is used to generate the ground truth Image compressions (also known as "reference standard") from multiple manual delineations prepared by multiple experts.

\section{Literature Survey}

Greeshma Gopal, Dr E.Grace Mary Kanaga (2013) Breast cancer is a public health problem. A tumour can be of two types benign or malignant. In benign tumours the cells are normal in appearance, but it is not cancerous, the cells will grow slowly but it does not spread to other parts of the body. But malignant tumours can spread to other parts of the body, and it is cancerous. These diseases mostly [14] occur in women, but men can get it, too. The most challenging area in medical imaging is image compression. In image compression the low-energy-X-rays is used to create images and to examine the human breast and thus it helps to detect the breast cancer at the early stage by detecting the small calcium deposits.

Shapiro, Linda G. \& Stockman, George C. (2002) [20] after being compressioned, the image or the mass lesion region can be further used by physicians, helping them to take decisions that involve their patients' health.

Commowick O, Warfield SK. (2010) MAP-based formulation of the STAPLE algorithm is used previously, for a different purpose, in order to merge the manual delineations made by multiple experts [11], [10]; it is used in the context of performing fusion with missing manual delineations for some of the structures of interest, in one or more template image. Such situation arises when some of the experts did not delineate all the structure of interest but delineated only a subset of all the labels.

Commowick O, Akhondi-Asl A, Warfield SK. (2012) proposed to incorporate this "missing" information into the STAPLE by appropriately constraining the performance parameters through the MAP formulation [11], [10]. The approach is specifically designed to deal with the fusion problem in the presence of missing data. The current manuscript addresses a completely different problem of learning prior knowledge about the performance parameters of automated Image compressions obtained from multiple template images.

\section{A. Image compression:}

\section{Methodology}

The image compression is the best system for an early analysis of the bosom malignancy. Finding an exact and productive bosom locale division strategy still [4] remains a testing issue in computerized image compression. imagedivision as a rule includes ordering mammograms into a few unmistakable areas, including the bosom fringe [9], the areola [8] with the pectoral muscle. The imperative part on an image is the bosom periphery, likewise, called the skin-air interface, or chest restrict. The chest shape can be gotten by allocating the imageinto bosom and non-chest districts.

\section{B. Proposed Methodology:}

There have been different methodologies proposed to the undertaking of dividing the breast profile area in mammograms.

\section{(i) Local Map View:}

Let $\mathbf{M}=\left\{\mathbf{M}_{1}, . ., \mathbf{M}_{\mathbf{i}}, \ldots, \mathbf{M}_{\mathbf{N}}\right\}$ be a matrix of size $\mathbf{K} \times \mathbf{N}$, where $\mathrm{K}$ and $\mathrm{N}$ are respectively the [10] values of image and the principle of voxels. In this matrix, $\mathbf{M}_{\mathbf{i}}=\left[\mathbf{M}_{\mathbf{i 1}}, \ldots, \mathbf{M}_{\mathbf{i j}}, \ldots, \mathbf{M}_{\mathbf{i J}}\right]^{\prime}$ and $\mathbf{M}_{\mathbf{i j}}$ is the label of the template $\mathbf{j}$ at voxel $\mathrm{i}$. The goal here is to estimate the output Image compression $\mathbf{K}=\left\{\mathbf{K}_{1}, \ldots, \mathbf{K}_{\mathbf{i}}, \ldots, \mathbf{K}_{\mathrm{N}}\right\}$ and the performance parameters $\boldsymbol{\theta}=\left\{\boldsymbol{\theta}_{1}, \ldots, \boldsymbol{\theta}_{\mathrm{j}}, \ldots, \boldsymbol{\theta}_{\mathbf{J}}\right\}$ where $\theta \mathrm{j}$ is the matrix of size $\mathbf{S} \times \mathbf{S}, \boldsymbol{\theta}_{\mathrm{js}}{ }^{\prime} \mathbf{S}=\mathbf{f}\left(\mathbf{M}_{\mathrm{ij}}=\mathbf{s}^{\prime} \mid \mathbf{K}_{\mathrm{i}}\right.$ $=\mathbf{s})$, and $\mathrm{S}$ is the number of Image compression labels. 
The following LVM algorithm demonstrates the Image compression of the images with the various Image compression and clustering possibilities with the images that is to be reached in the way to its boundary and its specified region in the mapping and its consistency to the various indexes and edge with the unique specification of images.

\section{Aspects of Proposed Algorithm:}

- Compression the images on basis of its functions with the clustering possibilities.

- This allows the user to choose instances for detailed analysis and filter the outlier instances from the input, next specific feature selection process identifies regions with systematic characteristics across the images.

\section{INPUT: The normal original image}

OUTPUT: Compressed images

STEP: 1 for all images Mi from initial boundary to destination boundary

//images which is to be compressioned by the boundary section//

STEP: 2 while $M_{i}$ has exactly one edge do

edge e $=k$. edge $(\mathrm{Ke})$;

e. level = k. map;

detect edge e;

//the image accessing for edge detection by the various sub region//

STEP: 3 if ( $D_{i j}$ is not the connected region)

$M_{i}-M_{i j}>\left(e_{i .}\right.$ edge level)

// the connected sub region by its feature//

then

STEP:4 View the sub region of edge map $e_{1}$;

//neglecting the intra connected sub region//

else

e. boundary $=\min ($ e. region $+1-\tau$, e.edge level $)$;

// update the new boundary region //

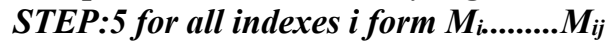

// the indexes of the boundary subset

region //

STEP: 6 while $n$ has exactly one child do

Initial $M_{i}=M_{i j .} \operatorname{map}(e)$;

e. edge= b. region;

STEP: 7 end.

\section{delete unmatched sub region; \\ Correct point map $\left(M_{i, e}\right)$;}

(ii) Staple Algorithm:

STAPLE is clear to apply to clinical imaging information, it promptly empowers appraisal of the execution of a computerized [21] picture division calculation and empowers coordinate correlation of human rater and calculation execution. This calculation considers an accumulation of divisions and registers a probabilistic gauge of the genuine division [21] and a proportion of the execution level spoken to by particular division.

$$
N i=K\left[\frac{1}{2} \sum_{i}(\operatorname{Mi}(n))^{2}\right]
$$

// the above equation states the principle of $n$ values of image in frequency range//

$$
\left.K=\left[\frac{1}{2} \sum_{i} p_{i}(n)-q i(n)\right)^{2}\right]
$$

// the constant value $k$ with the high

efficiency rating with more prominence//

$$
M i j=\frac{1}{k+1} \sum_{N=0}^{k}[d(M)-M i j(N)]^{2}
$$

//Where Mij is the matrix of stimulus truth 


\section{performance level estimation with the}

both column and the row vector//

Let M1................Mn be the column vector and

K1...................Kn be the row vector

$[M x K]^{T}=\max M i \ldots \ldots . . M n / K i \ldots . . . K n$

$\operatorname{Max} K i \ldots \ldots n=k[K i / k i j]$

// the frequency of the matrix with its higher

efficiency on both rows and columns//

$\theta i=[\theta i / \theta i j]^{T}$ implies values for the row and the column vector.

$$
\partial j=[\theta i / \theta i j] T \quad \ldots \ldots \ldots \ldots[\theta n / \theta n j] T
$$

// it finally shows both the column and the row Function delicate with each other's/

\section{Result and Discussions}

The limit of STAPLE to precisely appraise the genuine division, even within the sight of a dominant part of ratters creating related mistakes, was illustrated. The quantitative appraisal of compression of tissues from MRI of a breast was completed keeping in mind the end prospect to show the access of STAPLE with 3-D volumetric unordered category information's. Observational planning tests were likewise completed to show the reasonableness of the estimation plot.

\section{Efficiency Comparison:}

Table.1. Accuracy Rate of LMV with Region

\begin{tabular}{|c|l|l|l|l|l|}
\hline Methods & Values & Values & Values & Values & Values \\
\hline SIFT & 50 & 48 & 48 & 50 & 48 \\
\hline K-means & 52 & 52 & 53 & 51 & 52 \\
\hline Region Grow & 46 & 46 & 48 & 46 & 48 \\
\hline LMV & 53 & 54 & 53 & 55 & 54 \\
\hline
\end{tabular}




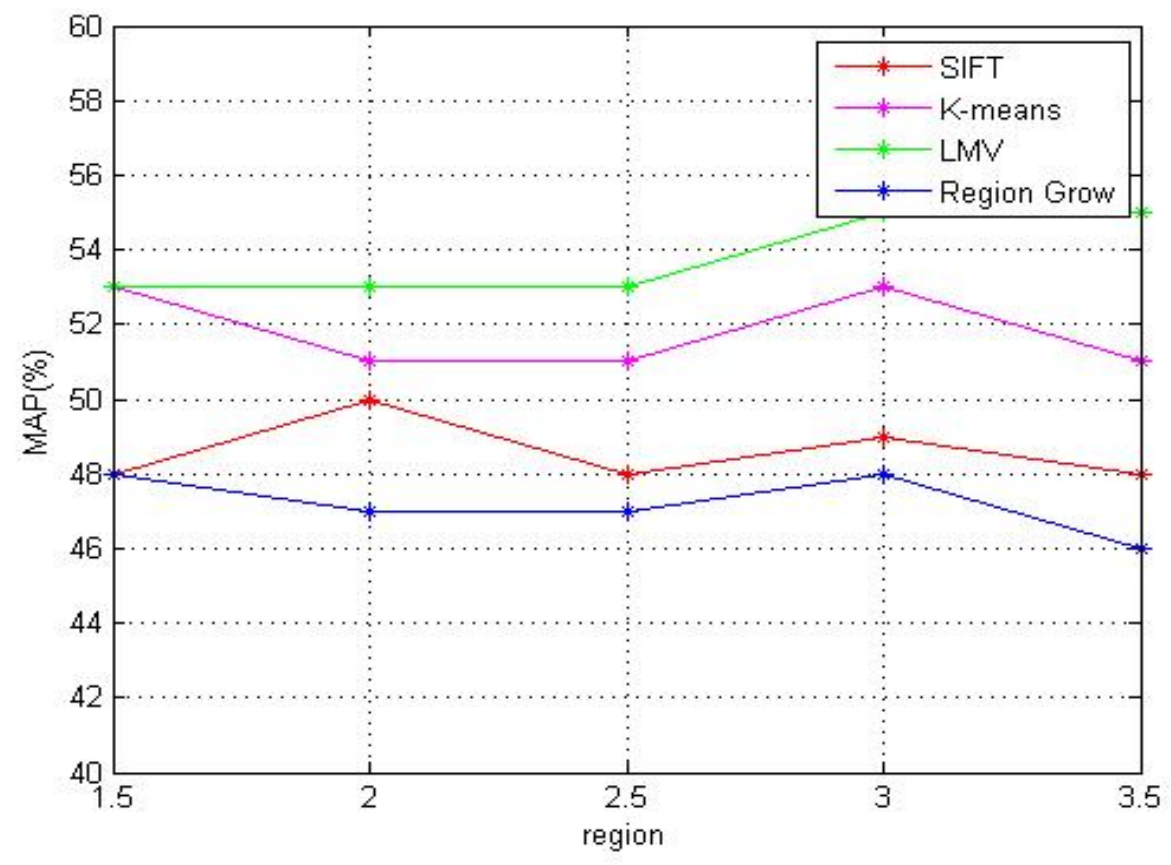

Fig.1. Efficient analysis of LMV with Region.

The Fig.1. explains the efficiency comparison of proposed LMV technique when comparing the Mapping Accuracy with existing 3 Image compression algorithm SIFT $=$ Scale invariant feature transform (This $\mathrm{s}$ used to extract the features of given data)K-means $=$ one of the clustering technique (this $\mathrm{s}$ used for grouping and classifying the groupwise data's)Region Grow= Tumor areas growth and Image compression purpose (Initial to ending region identification is possible in this).In this the proposed algorithm is accurately compressioning abnormal when the number of cluster region varies.

Table.2. Accuracy Rate of LMV with Weight

\begin{tabular}{|c|l|l|l|l|}
\hline Methods & Values & Values & Values & Values \\
\hline SIFT & 50 & 48 & 48 & 48 \\
\hline K-means & 54 & 54 & 53 & 54 \\
\hline Region Grow & 48 & & & \\
\hline LMV & 57 & 48 & 48 & 47 \\
\hline
\end{tabular}




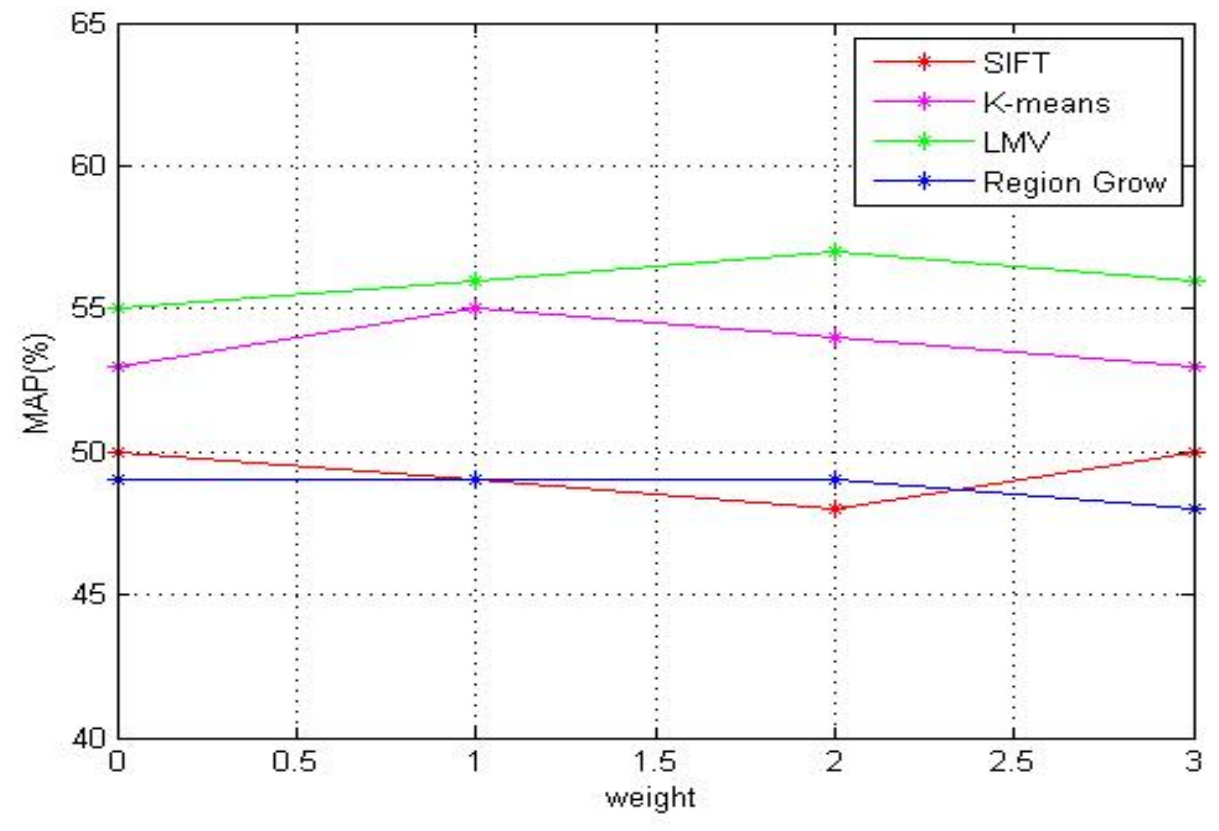

Fig.2. Efficient analysis of LMV with Weight

The Fig.2.explains the efficiency comparison of our proposed LMV Technique on basis of the region when comparing the Mapping Accuracy with existing 3 Image compression algorithm SIFT= Scale invariant feature transform (This s used to extract the features of given data)K-means $=$ one of the clustering technique (this $\mathrm{s}$ used for grouping and classifying the groupwise data's)Region Grow= Tumor areas growth and Image compression purpose (Initial to ending region identification is possible in this). On comparing both the system the proposed technique shows the higher efficiency on the weight than the other process.

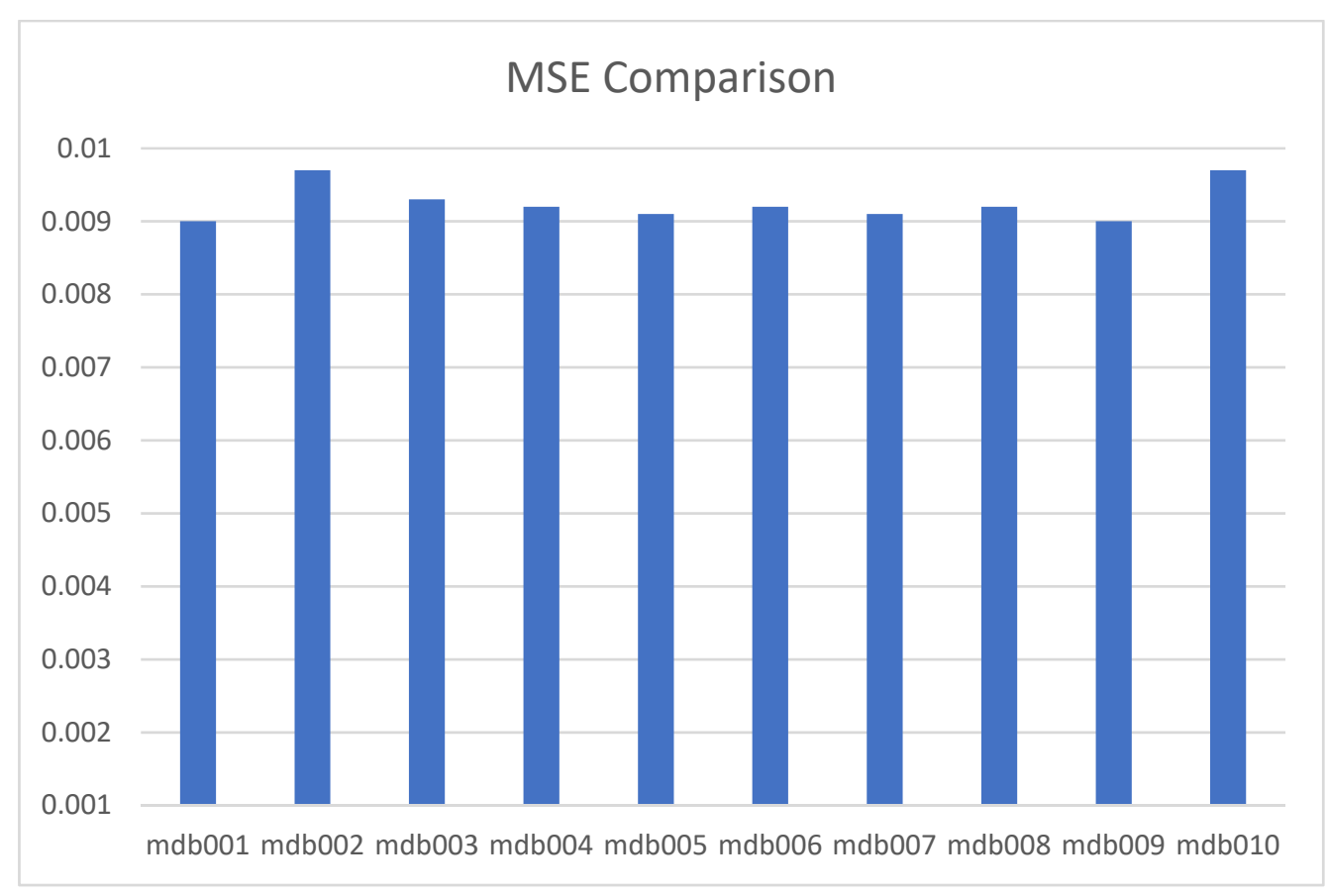

Fig.3. MSE comparison

The Fig.3. analysis shows the Mean Square Error (MSE) which is used to predict the error value that is a variation between the estimated value and the actual value obtained whereas the MSE represents the cumulative squared error between the compressed and the original image as the $\mathrm{X}$ axis= name of the images and $\mathrm{Y}$ axis $=$ Percentage in units 


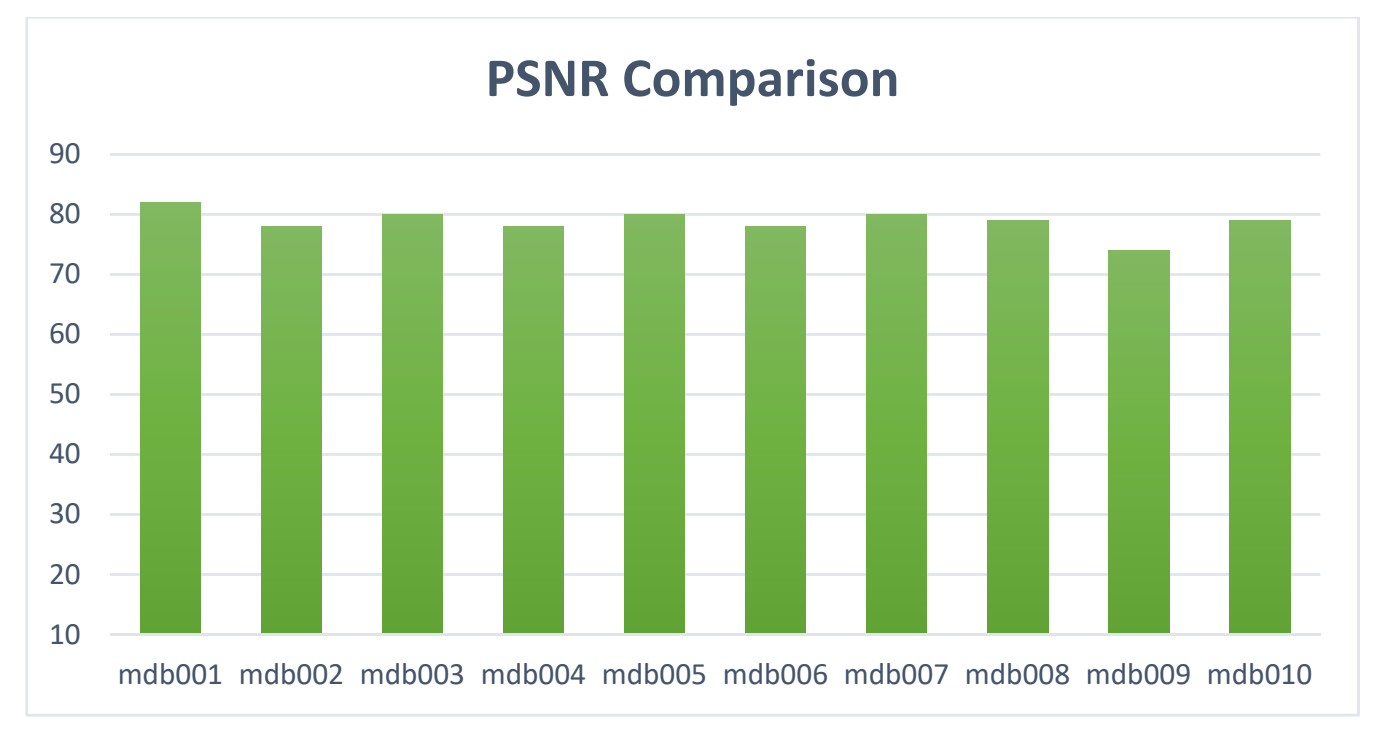

Fig.4. PSNR comparison

From the Fig.4., it is seen that the correlation metric is registered among the pre-handling strategies regarding PSNR metric. In x-hub the calculations are considered and in y-pivot the PSNR esteem is considered. The proposed LMV calculation gives higher PSNR esteem when contrasted with other technique The PSNR block computes the peak signal-to-noise ratio, in decibels, between two images, whereas PSNR represents a measure of the peak error. The outcome affirms that the proposed framework increases more prominent discourse acknowledgment results whereas $\mathrm{X}$ axis $=$ name of the images, $\mathrm{Y}$ axis $=$ Percentage in units.

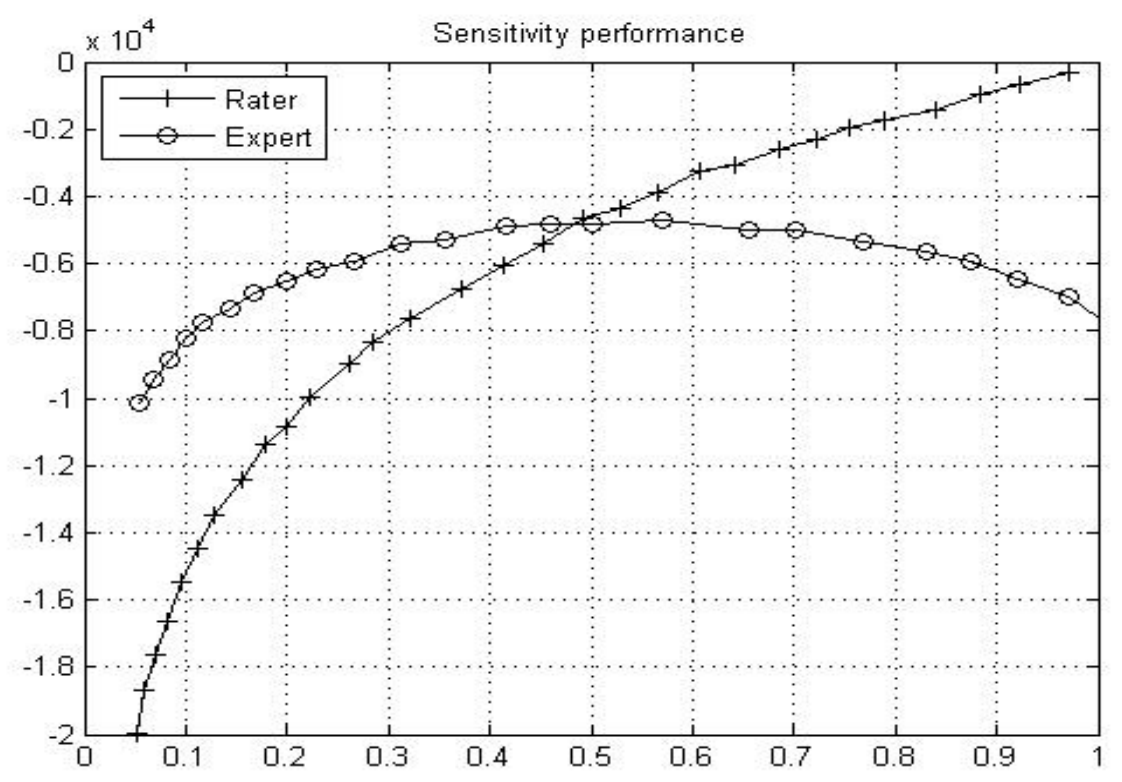

Fig.5. Sensitivity Performance

The Fig.5. clarifies the affectability execution of the picture in a staple correlation framework forms whereas diagram demonstrates the affectability execution of both rater and the master. 


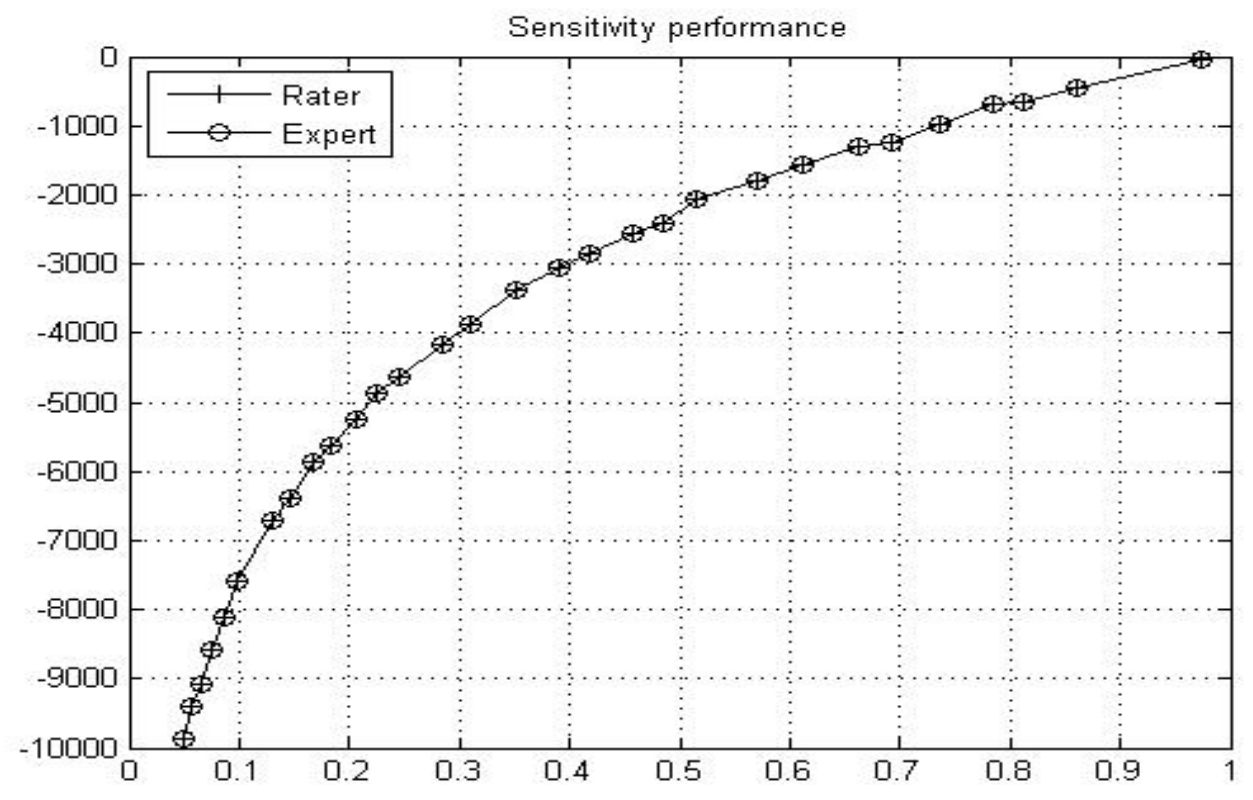

Fig.6. Sensitivity Performance of the Expert

The Fig.6. clarifies the affectability execution of the picture in a staple examination framework forms. This diagram demonstrates the affectability execution of and the master with the high productivity of the execution level.

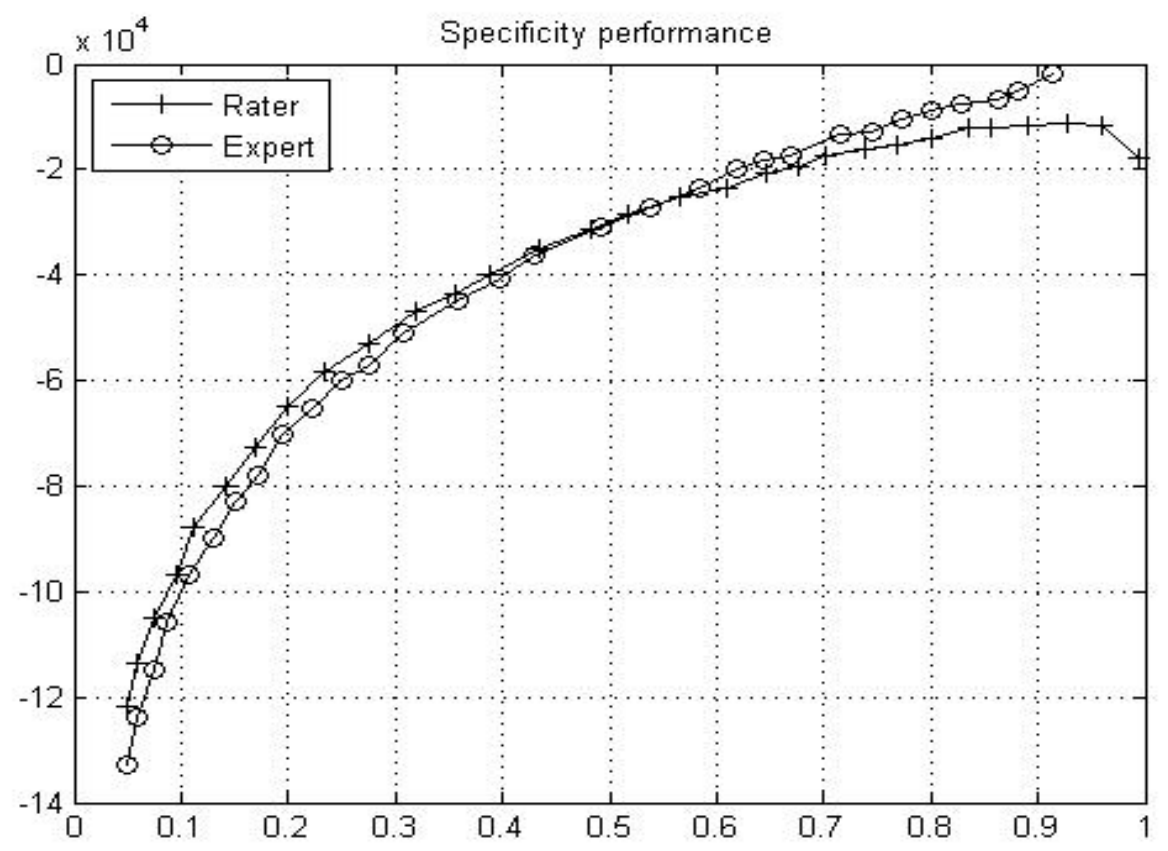

Fig.7. Specificity Performance

The Fig.7. clarifies the affectability execution of the picture in a staple examination framework form. This diagram demonstrates the specificity execution of both rater and the master. 


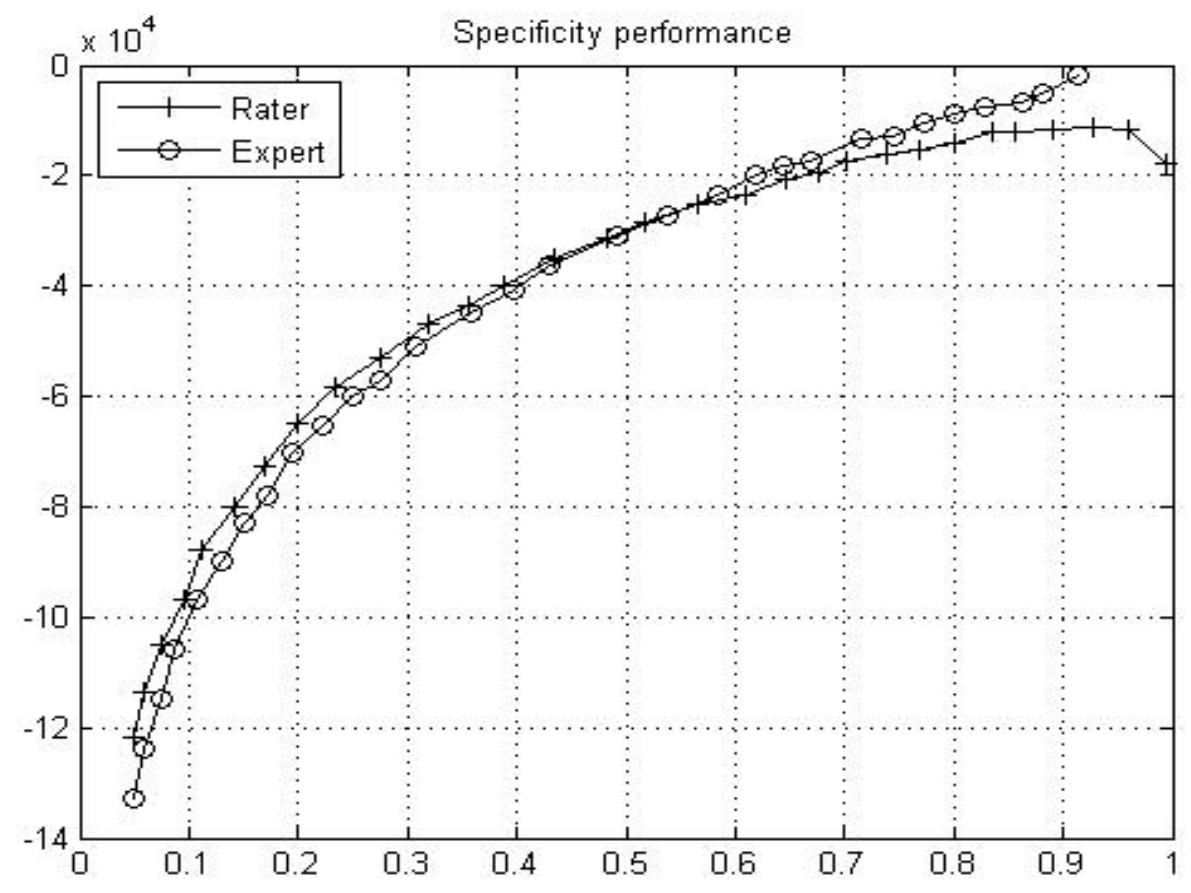

Fig.8. Specificity Performance of Expert

The Fig.8. clarifies the specificity staple examination framework forms whereas the diagram demonstrates the affectability execution of and the master with the high proficiency of the execution level.

\section{Conclusion}

The proposed technique exhibited a calculation for taking a gathering of both parallel and unordered multi classification divisions and all the while building a gauge of the shrouded genuine division and a gauge of the execution [21] level of every division generator. This can be utilized to describe any kind of division generator, including new division calculations or human administrators, by guide correlation with the assessed true Image compression. By this, In Future it may incorporate by consolidating this strategy into other efficient division calculations and methods with the vitality utilitarian weights naturally by means of preparing information about Image compression.

\section{Acknowledgement}

Extremely thankful to Anna Nagar X-rays \& Diagnostic Centre, Chennai for giving various images for research purpose. It is also helpful to test algorithm under various parameters.

\section{References}

[1] Abhishek Choudhary, Manasvi Mannan (2016) "Mammographic Image Enhancement analysis using Fuzzy Logic" International Journal of Advance Engineering and Research Development Volume 3, Issue 2, p.p:6-7.

[2] Akhondi-Asl A, Hoyte L, Lockhart M, Warfield S. (2014) "A logarithmic opinion pool based STAPLE algorithm for the fusion of Image compressions with associated reliability weights". IEEE Transactions on Medical Imaging.; 33(10) p.p:1997-2009.

[3] Akhondi-Asl A, Warfield S. (2013) "Simultaneous truth and performance level estimation through fusion of probabilistic Image compressions." IEEE Transactions on Medical Imaging. 32(10): p.p:1840-1852.

[4] Armen Sahakyan, Hakop Sarukhanyan (2012) "Image compression of the Breast Region in Digital Mammograms and Detection of Masses” (IJACSA) International Journal of Advanced Computer Science and Applications, Vol. 3, No.2, p.p:102-103.

[5] Artaechevarria X, Munoz-Barrutia (2009) "A. Combination strategies in multi-atlas image Image compression: Application to brain MR data". IEEE Transactions on Medical Imaging. 28(8):1266-1277

[6] Asman A, Landman B. (2012); "Formulating spatially varying performance in the statistical fusion framework". IEEE Transactions on Medical Imaging. 2012; 31(6): p.p:1326-1336.

[7] Cardoso M, Leung K, Modat M, Barnes J, Ourselin S. (2011) "Locally ranked staple for template based Image compression propagation". Workshop on Multi-Atlas Labeling and Statistical Fusion.

[8] Chandrasekhar R, and Y. Attikiouzel, 1997. "A Simple Method for Automatically Locating the Nipple on Mammograms", IEEE Transactions on Medical Imaging, vol. 16, pp. 483-494

[9] Chandrasekhar,R and Y. Attikiouzel, "Automatic Breast Border Image compression by Background Modelling and Subtraction", 5th International Workshop on Digital image compression (IWDM), (Yaffe M. ed.), Medical Physics Publishing, Madison, USA, pp. $560-565$.

[10] Commowick O, Akhondi-Asl A, Warfield SK. (2012) "Estimating a reference standard Image compression with spatially varying performance parameters: Local MAP STAPLE.” IEEE Transactions on Medical Imaging. 31(8) p.p:1593-1606. 
[11] Commowick O, Warfield SK. (2010) "Incorporating priors on expert performance parameters for Image compression validation and label fusion: a Maximum A Posteriori STAPLE" Medical Image Computing and Computer-Assisted Intervention (MICCAI). 6363: p.p:25-32

[12] Domingos P and M. J. Pazzani, (1997). "On the optimality of the simple bayesian classifier under zero-one loss," Machine Learning 29(2-3), pp. 103-130, 1997.

[13] Gorthi S, Bach Cuadra M, Tercier P-A, Allal A, Thiran J-P. (2013) "Weighted shape-based averaging with neighborhood prior model for multiple atlas fusion-based medical image Image compression”. IEEE Signal Processing Letters. 20(11): p.p:1034-1037.

[14] Greeshma Gopal, Dr E.Grace Mary Kanaga(2013) "A Study on Enhancement Techniques for images" International Journal of Advanced Research in Electronics and Communication Engineering (IJARECE) Volume 2, Issue 1,

[15] Indra Kanta Maitra, Sanjay Nag, Samir K Bandyopadhyay (2011) "Accurate Breast Contour Detection Algorithms in Digital Mammogram" International Journal of Computer Applications (0975 - 8887) p.p:6-9.

[16] Michael A. Wirth, Alexei Stapinski, "Image compression of the breast region in mammograms using active Contours" Dept. of Computing and Information Science, University of Guelph.

[17] Neapolitan R, (2003) "Learning Bayesian Networks", Prentice Hall, Upper Saddle River, NJ.

[18] Rohlfing T, Maurer JCR. (2007) "Shape-based averaging". IEEE Transactions on Image Processing. 16(1): p.p:153-161.

[19] Sabuncu M, Yeo B, Van Leemput K, Fischl B, Golland P. (2010) "A generative model for image Image compression based on label fusion". IEEE Transactions on MedicalImaging.;29(99: p.p:1714-1729.

[20] Shapiro, Linda G. \& Stockman, George C. (2002). "Computer Vision". Prentice Hall. ISBN 0-13-030796-3.

[21] Simon K. Warfield,Kelly H. Zou, and William M. Wells(2014) "Simultaneous Truth and Performance Level Estimation (STAPLE): An Algorithm for the Validation of Image Image compression" IEEE Trans Med Imaging. Author manuscript; available in PMC 2005 November 14 p.p:903-921.

[22] Smola A.J and B. Scholkopf, (2004) "A tutorial on support vector regression," Statistics and Computing 14(3), pp. 199-222,

[23] Subrahmanyam Gorthi, Alireza Akhondi-Asl, and Simon K. Warfield (2015) "Optimal MAP Parameters Estimation in STAPLE using Local Intensity Similarity Information” IEEE J Biomed Health Inform.; 19(5): p.p:1589-1597.

[24] Vercauteren T, Pennec X, Perchant A, Ayache N. (2007) "Non-parametric differomorphic image registration with the demons algorithm". Medical Image Computing and Computer-Assisted Intervention-MICCAI p.p:319-326.

[25] Wang H, Suh J, Das S, Pluta J, Craige C, Yushkevich P. (2013) "Multi-atlas Image compression with joint label fusion". IEEE Transactions on Pattern Analysis and Machine Intelligence. ;35(3): p.p:611-623.

[26] Warfield S, Zou K, Wells W. (2004) "Simultaneous truth and performance level estimation (STAPLE): an algorithm for the validation of image Image compression". IEEE Transactions on Medical Imaging.; 23(7): p.p:903-921.

\section{Authors Profile}

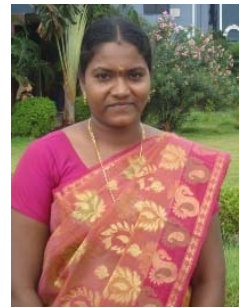

Rajeswari Chellathurai received her MCA in Madurai Kamaraj University and M.Phil. (CS) in SCSVMV University Kanchipuram, TN, India. She is currently pursuing $\mathrm{Ph}$. D in SCSVMV University, Kanchipuram. She is working as Assistant Professor, Dept of Computer Science, Soka Ikeda College of Arts \& Science for Women, Chennai, TN, India. She has qualified TN SET. Her research interests are Image Processing, Image Compression and Data Mining. She has presented and participated more papers and workshops in various colleges. She published many journals in National and International Publications.

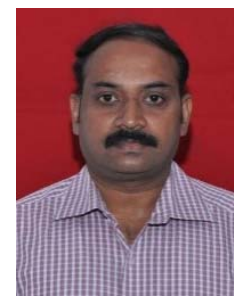

Dr.S.Prakasam working as Associate Professor, Dept of CSA, SCSVMV University, Kanchipuram, TN, India. He has more than 20 years experience. His research interests are Software Engineering, Data Mining, Data Communication \& Networks. He has presented papers in various national and international conferences. He published many journals in international publications. 